\title{
Correction: Building a Digital Tool for the Adoption of the World Health Organization's Antenatal Care Recommendations: Methodological Intersection of Evidence, Clinical Logic, and Digital Technology
}

Samira M Haddad ${ }^{1,2}$, MSc, MD, PhD; Renato T Souza ${ }^{1,2}$, MSc, MD, PhD; Jose Guilherme Cecatti ${ }^{1,2}$, MSc, MD, PhD; Maria Barreix ${ }^{3}$, MHS; Tigest Tamrat ${ }^{3}$, MPH; Carolyn Footitt ${ }^{4}$, MSPH; Garrett L Mehl ${ }^{3}$, PhD; Inraini F Syah ${ }^{5}$, MPH; Anuraj H Shankar ${ }^{5,6,7}$, PhD; Özge Tunçalp ${ }^{3}, \mathrm{MD}, \mathrm{PhD}$

\footnotetext{
${ }^{1}$ Department of Obstetrics and Gynecology, School of Medical Sciences, University of Campinas, Campinas, Brazil

${ }^{2}$ Center for Research in Reproductive Health of Campinas (CEMICAMP), Campinas, Brazil

${ }^{3}$ UNDP-UNFPA-UNICEF-WHO-World Bank Special Programme of Research, Development and Research Training in Human Reproduction (HRP), Department of Reproductive Health and Research, World Health Organization, Geneva, Switzerland

${ }^{4}$ Ona Systems Inc, Nairobi, Kenya

${ }^{5}$ Summit Institute of Development, Mataram, Indonesia

${ }^{6}$ Centre for Tropical Medicine and Global Health, Nuffield Department of Medicine, University of Oxford, Oxford, United Kingdom

${ }^{7}$ Eijkman-Oxford Clinical Research Unit, Eijkman Institute for Molecular Biology, Jakarta, Indonesia
}

\section{Corresponding Author:}

Jose Guilherme Cecatti, MSc, MD, PhD

Department of Obstetrics and Gynecology

School of Medical Sciences

University of Campinas

101 Alexander Fleming Street

University of Campinas

Campinas, 13083-891

Brazil

Phone: 551935219482

Email: cecatti@unicamp.br

\section{Related Article:}

Correction of: https://www.jmir.org/2020/10/e16355

(J Med Internet Res 2020;22(10):e24891) doi: 10.2196/24891

In "Building a Digital Tool for the Adoption of the World Health Organization's Antenatal Care Recommendations: Methodological Intersection of Evidence, Clinical Logic, and Digital Technology" (J Med Internet Res 2020;22(10):e16355) the authors noted three errors.

The name of author Tigest Tamrat was incorrectly listed as "Tigist Tamrat". This has now been changed to the correct spelling.
Under the section "Structured Documentation", one instance of the phrase "business process mapping (BPM)" has been corrected to "business process mapping notation (BPMN)".

Figure 6 has been replaced with a new version of subsection B (seen below), as the authors did not have permission to use images displayed in the originally published version of subsection B. Figure 6 subsections A, C, and D have not been changed from the originally published version. 
Figure 6. Screenshots of the World Health Organization Digital Antenatal Care (WHO digital ANC) module. A: List of patients; B: Individual patient record summary; C: Home screen; D: Patient contact summary.

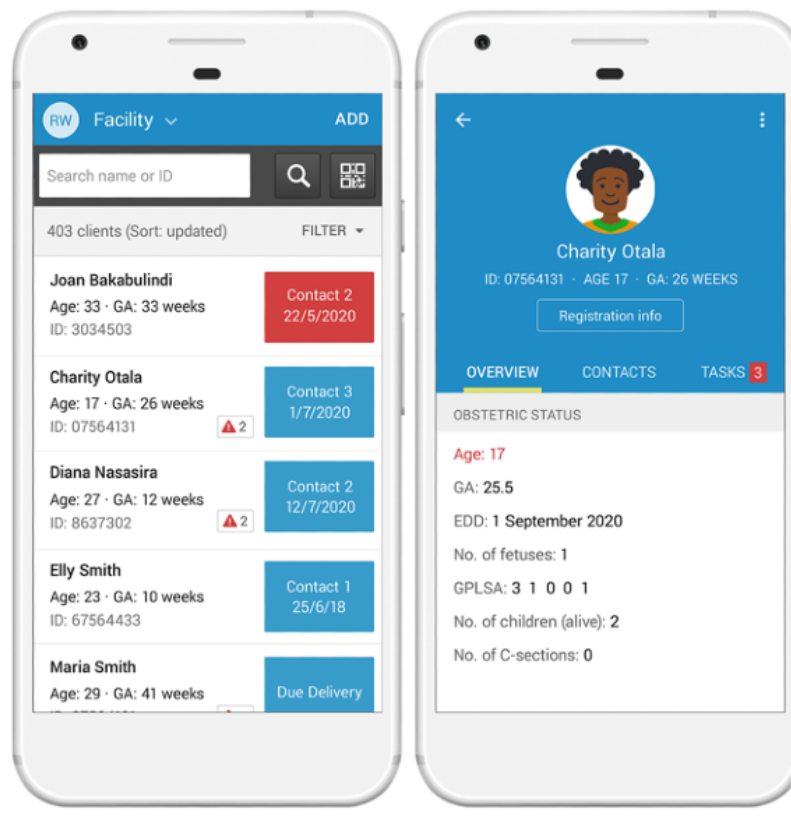

The correction will appear in the online version of the paper on the JMIR Publications website on October 13, 2020, together with the publication of this correction notice. Because this was

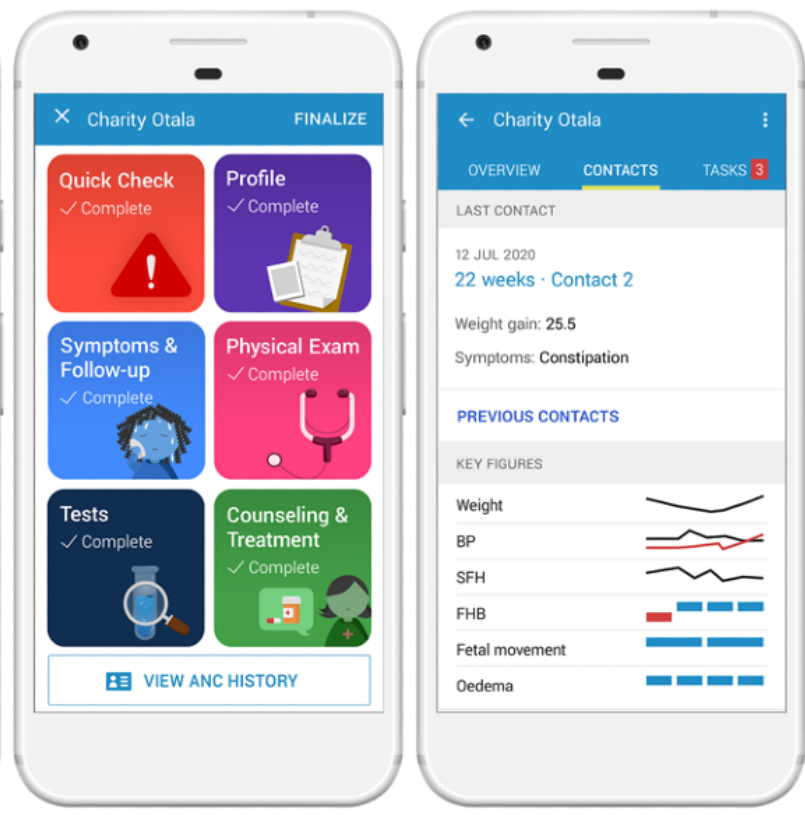

made after submission to PubMed, PubMed Central, and other full-text repositories, the corrected article has also been resubmitted to those repositories.

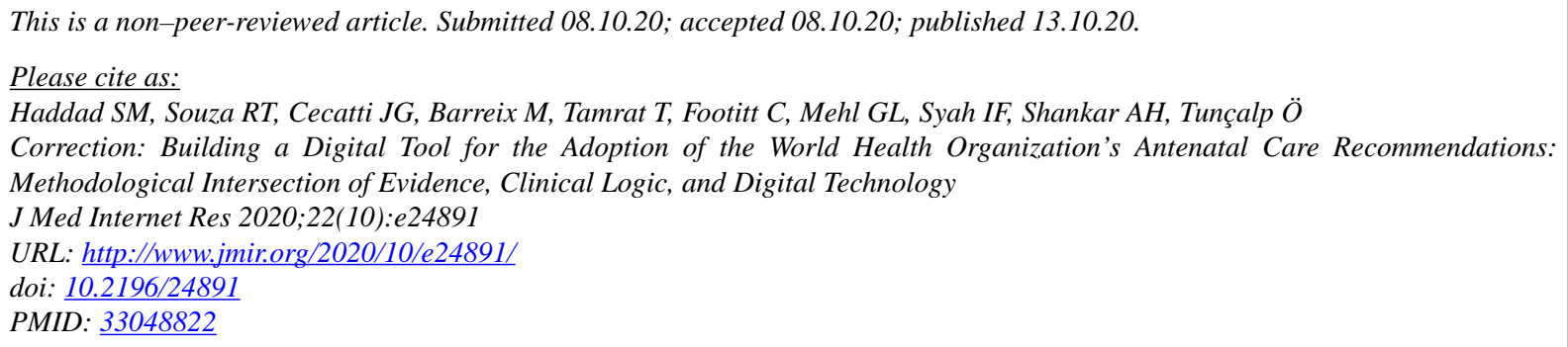

(C)Samira M Haddad, Renato T Souza, Jose Guilherme Cecatti, Maria Barreix, Tigest Tamrat, Carolyn Footitt, Garrett L Mehl, Inraini F Syah, Anuraj H Shankar, Özge Tunçalp. Originally published in the Journal of Medical Internet Research (http://www.jmir.org), 13.10.2020. This is an open-access article distributed under the terms of the Creative Commons Attribution License (https://creativecommons.org/licenses/by/4.0/), which permits unrestricted use, distribution, and reproduction in any medium, provided the original work, first published in the Journal of Medical Internet Research, is properly cited. The complete bibliographic information, a link to the original publication on http://www.jmir.org/, as well as this copyright and license information must be included. 